\title{
Celiac disease and hearing loss
}

\author{
Lemajic-Komazec $S^{1}$, Abenavoli L ${ }^{1,2}$ \\ Clinical Center of Vojvodina, Faculty of Medicine, University of Novi Sad, \\ Novi Sad, Serbia. l.abenavoli@unicz.it
}

Dear Sir,

Celiac disease (CD) is an autoimmune gluten-dependent enteropathy, characterized by flat intestinal mucosa. Classical manifestation of CD includes malabsorption with impaired nutritional status, diarrhoea, abdominal distension and weight loss (1). CD can be associated with several extra-intestinal manifestations. Among them, neurological disorders have been reported, including epilepsy, peripheral neuropathy, myoclonus and posterior column demyelization, headache, cerebellar ataxia, brain atrophy and dementia (2). The etiopathogenesis of neurological manifestations in CD patients remains unclear, although brain vasculitis, the presence of anti-neuronal antibodies, the regional cerebral hypoperfusion and nutritional deficiencies, have been proposed as possible pathogenetic mechanisms (3). Hearing loss (HL) represents a common disorder in general population and the 2 major forms are conductive and sensorineural disorders. The prevalence of HL rises with age and in particular, HL affects about $20 \%$ of population aged from 48 to 59 years and between $25-40 \%$ of the population aged to 65 years and above (4).

We have read with a great interest the interesting article published by Suchan et al on the involvement of the immune response in the etiopathogenesis of some cochleovestibular disorders (5). An immune-mediated mechanism could be on the basis of both, HL and the CD related neurological disorders, an association between CD and HL could be possible. In fact, immune-mediated HL is a clinical entity largely discussed and described in literature, and several immune-mediated mechanisms including autoantibodies, autoreactive T cells, and immune-complex depositions have been explored as possible mechanisms on that basis of HL (5). However, another pathogentic link between HL and CD can be represented by malnutrition and in particular low iron blood concentration and its effects on vasculature and nervous system. It is disputable whether cochlear system is highly susceptible to ischemic damage subsequently to iron deficiency anemia, since only the labyrinthine artery supplies blood into this anatomic area.

Further studies in a larger sample-size also investigating immunological parameters as a ear related or other anti-neuronal antibodies and nutritional impairments, are needed to better understand this association. Our opinion is to recommend a screening by audiometric examinations in CD patients, in particular in childhood considering the high risk of irreversible deficits in speech, language, cognitive and psychosocial development and for recognizing hearing loss early during the course of the disease.

\section{References}

1. Ludvigsson JF, Leffler DA, Bai JC et al. The Oslo definitions for coeliac disease and related terms. Gut 2013; 62 (1): 43-52.

2. Casella G, Bordo BM, Schalling R et al. Neurological disorders and celiac disease. Minerva Gastroenterol Dietol 2016; 62 (2): 197-206.

3. Abenavoli L. Brain hypoperfusion and neurological symptoms in celiac disease. Mov Disord 2010; 25 (6): 799-800.

4. Leggio L, Cadoni G, D’Angelo C et al. Coeliac disease and hearing loss: preliminary data on a new possible association. Scand J Gastroenterol 2007; 42 (10): 1209-1213.

5. Suchan M, Kaliarik L, Krempaska S et al. Immune-mediated cochleovestibular disease. Bratisl Lek Listy 2016; 117 (2): 87-90.

${ }^{1}$ Clinical Center of Vojvodina, Faculty of Medicine, University of Novi Sad, Novi Sad, Serbia, and ${ }^{2}$ Department of Health Sciences, University "Magna Græcia”, Catanzaro, Italy

Address for correspondence: L. Abenavoli, MD, PhD, Department of Health Sciences, University Magna Graecia, Viale Europa, 88100 Catanzaro, Italy.

Phone: +39.0961.3694387, Fax: +39.0961.754220 